\title{
Influence of fluctuating strain on exciton reflection spectra
}

\author{
Skettrup, Torben
}

Published in:

Physical Review B

Link to article, DOI:

10.1103/PhysRevB.25.984

Publication date:

1982

Document Version

Publisher's PDF, also known as Version of record

Link back to DTU Orbit

Citation $(A P A)$ :

Skettrup, T. (1982). Influence of fluctuating strain on exciton reflection spectra. Physical Review B, 25(2), 984990. https://doi.org/10.1103/PhysRevB.25.984

\section{General rights}

Copyright and moral rights for the publications made accessible in the public portal are retained by the authors and/or other copyright owners and it is a condition of accessing publications that users recognise and abide by the legal requirements associated with these rights.

- Users may download and print one copy of any publication from the public portal for the purpose of private study or research.

- You may not further distribute the material or use it for any profit-making activity or commercial gain

- You may freely distribute the URL identifying the publication in the public portal

If you believe that this document breaches copyright please contact us providing details, and we will remove access to the work immediately and investigate your claim. 


\title{
Influence of fluctuating strain on exciton reflection spectra
}

\author{
T. Skettrup \\ Physics Laboratory III, Technical University of Denmark, DK-2800 Lyngby, Denmark
}

(Received 11 February 1981)

The influence of an internal distribution of strain on the exciton reflection spectra is investigated. The resulting fluctuating optical constants give rise to a fluctuating phase of reflectivity. The standard deviation $\sigma$ of these phase fluctuations is the quantity which can be observed, for example, between crossed polarizers or from ellipsometric measurements. Assuming the phase fluctuations to obey a Gaussian distribution, $\sigma$ can be expressed in a simple way in terms of the degree of polarization or the depolarization of the reflected light. $\sigma$ is then derived in terms of the standard deviations of the fluctuating strain components for wurtzite-structure crystals. To do this it is necessary to find the behavior of the six independent (complex) photoelastic coefficients in the excitonic resonance region. A simple approximation makes it possible to obtain these. Furthermore, it is necessary to derive the dependence of the phase of reflectivity on the direction of the fluctuating optical axis. The results obtained for $\sigma$ are compared with the experimental depolarization spectra of $\mathrm{ZnO}$. The only fitting parameter is the common standard deviation of the strain components. It is found that the fluctuating strain can reproduce the positions and relative magnitudes of the resonant depolarization peaks, but fails to reproduce the background level of depolarization and the magnitude of the $A$ peak in one geometry.

\section{INTRODUCTION}

Optical spectra like exciton spectra are usually computed for ideal and perfect crystals. In real crystals, however, there are several mechanisms causing nonideal behavior. These include vacancies, interstitials, dislocations, impurities and other imperfections like clusters, twins, etc. Furthermore, the surface is never completely planar, no matter how well it is polished or etched. In addition to these statical defects there exists the dynamical disorder due to lattice vibrations, important even at zero temperature.

One of the main effects of these imperfections is an internal distribution of local strains all over the crystal volume. The purpose of the present work is to investigate the influence of this local strain distribution on the exciton reflection spectra.

\section{DEPOLARIZATION TECHNIQUE}

The result of the internal strain distribution is that the optical constants are fluctuating in the crystal. This implies a reduced degree of coherence and an increased depolarization of the light that has interacted with the crystal. Since the depolarization is easier to measure than the degree of coherence, we shall concentrate on depolarization spectra in the present work. In Fig. 1 a typical ellipsometer setup is shown. If the incoming field has the magnitude $E_{0}$, the outgoing field has, before passing the analyzer 4 , the magnitude

$$
\begin{aligned}
\overrightarrow{\mathrm{E}}_{3}=E_{0}\left(\cos \beta a_{x} e^{i\left(\theta_{x}+\theta_{1}\right)} \hat{x}\right. \\
\left.+\sin \beta a_{y} e^{i\left(\theta_{y}+\theta_{2}\right)} \hat{y}\right),
\end{aligned}
$$

where $\hat{x}$ and $\hat{y}$ are unit vectors, $\beta$ is the angle between the polarizer and the $x z$ plane, and $a_{x} e^{i \theta_{x}}$ and $a_{y} e^{i \theta_{y}}$ are complex reflection (or transmission) coefficients. If the crystal is uniaxial its optical axis is directed along the $y$ axis. If the crystal is isotropic, then $a_{x}=a_{y}$ and $\theta_{x}=\theta_{y}$ in average, but they are fluctuating independently. $\theta_{1}$ and $\theta_{2}$ are the phase delays introduced by the compensator.

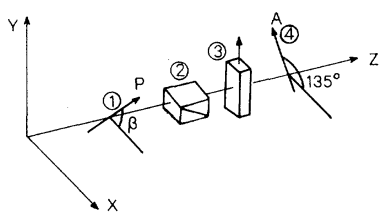

FIG. 1. Principle of ellipsometer. 1 Polarizer, 2 compensator, 3 sample, and 4 analyzer. 
Introducing now the coherency matrix ${ }^{1}$ we obtain

$$
\begin{aligned}
J & =\left\{\begin{array}{cc}
\left\langle E_{3 x} E_{3 x}^{*}\right\rangle & \left\langle E_{3 x} E_{3 y}^{*}\right\rangle \\
\left\langle E_{3 y} E_{3 x}^{*}\right\rangle & \left\langle E_{3 y} E_{3 y}^{*}\right\rangle
\end{array}\right\} \\
& =E_{0}^{2} a^{2}\left\{\begin{array}{cc}
1 & e^{-\sigma^{2} / 2} \\
e^{-\sigma^{2} / 2} & 1
\end{array}\right\},
\end{aligned}
$$

where \langle\rangle denotes the time average over long times, ${ }^{1}$ and

$$
a=a_{x} \cos \beta=a_{y} \sin \beta
$$

and

$$
\theta_{x}-\theta_{y}=\theta_{2}-\theta_{1}
$$

when the ellipsometer is adjusted for minimum transmitted light through the analyzer 4 . The evaluation of the averages of (2) proceeds as follows:

$$
\left\langle E_{3 x} E_{3 y}^{*}\right\rangle=E_{0}^{2} a_{0 x} a_{0 y}\left\langle\left(1+\frac{\Delta a_{x}}{a_{0 x}}\right)\left(1+\frac{\Delta a_{y}}{a_{0 y}}\right) e^{i \Delta \theta_{x y}}\right) e^{i\left(\theta_{0 x}-\theta_{0 y}-\theta_{2}+\theta_{1}\right)} \cos \beta \sin \beta,
$$

where only the fluctuating quantities contribute to the time average. In (5) we have

$$
\begin{aligned}
\Delta \theta_{x y} & =\theta_{x}-\theta_{0 x}-\left(\theta_{y}-\theta_{0 y}\right) \\
& =\Delta \theta_{x}-\Delta \theta_{y} .
\end{aligned}
$$

The time average of $\Delta a_{x}$ and $\Delta a_{y}$ must be zero, since it appears in first order in (5) and the amplitude is expected to fluctuate around the average amplitudes $a_{0 x}$ and $a_{0 y}$. Hence, only the phase fluctuations contribute in (5). The actual distribution function is unknown. However, in view of the large number of fluctuations assumed to be present, it seems reasonable to apply a Gaussian distribution

$$
P(\theta)=\left(2 \pi \sigma^{2}\right)^{-1 / 2} \exp \left[-\frac{\left(\theta-\theta_{0}\right)^{2}}{2 \sigma^{2}}\right),
$$

where $\sigma$ and $\theta_{0}$ are standard deviation and average value of the fluctuating phase. Hence, the time average of $(5)$ is equivalent to the average obtained by means of the distribution (7):

$\left\langle e^{i \Delta \theta_{x}}\right\rangle=\int_{-\infty}^{\infty} P\left(\theta_{x}\right) e^{i \Delta \theta_{x}} d \theta_{x}=e^{-\sigma_{x}^{2} / 2}$.

Then as shown in (2) we obtain

$$
\left\langle E_{3 x} E_{3 y}^{*}\right\rangle=E_{0}^{2} a^{2} e^{-\sigma^{2} / 2},
$$

where

$$
\sigma^{2}=\sigma_{x}^{2}+\sigma_{y}^{2}
$$

since (8) is independent of the sign of $\Delta \theta$.

The coherency matrix (2) can always be divided up into two matrices ${ }^{1}$ :

$$
J=\frac{1}{2} I_{d}\left\{\begin{array}{ll}
1 & 0 \\
0 & 1
\end{array}\right\}+\frac{1}{2} I_{p}\left\{\begin{array}{cc}
1 & \gamma \\
\gamma^{*} & 1
\end{array}\right\}
$$

where the first matrix describes completely unpolarized (depolarized) light with intensity $I_{d}$, and the second matrix describes completely polarized light with intensity $I_{p}$ when $|\gamma|=1$. Decomposing (2) according to (11) yields

$$
\begin{aligned}
& I_{d}=2 E_{0}^{2} a^{2}\left(1-e^{-\sigma^{2} / 2}\right), \\
& I_{p}=2 E_{0}^{2} a^{2} e^{-\sigma^{2} / 2} .
\end{aligned}
$$

Then the degrees of polarization of the light leaving the crystal in Fig. 1 is

$$
P=\frac{I_{p}}{I_{p}+I_{d}}=e^{-\sigma^{2} / 2}
$$

It is also convenient to introduce the depolarization (D) of the light as

$$
D=\frac{I_{d}}{I_{p}}=\frac{1-e^{-\sigma^{2} / 2}}{e^{-\sigma^{2} / 2}} \simeq \sigma^{2} / 2
$$

which is particularly useful when $\sigma^{2}<<1$.

Hence, by measuring the amount $I_{d}$ of depolarized light (i.e., the amount of light transmitted through the analyzer 4 in Fig. 1 when the ellipsometer is adjusted for minimum light transmission), the standard deviations of the fluctuating phases can be determined. In uniaxial crystals $\sigma_{x}$ and $\sigma_{y}$ usually have different spectral dependences so that it is possible to determine them separately.

\section{STRAIN FLUCTUATIONS}

In this section we shall derive $\sigma$ in terms of the standard deviations of the fluctuating strain components. To be more specific, we consider wurtzite structure since experimental results exist for $\mathrm{ZnO}$. It is convenient to use the photoelastic coefficients in 
this derivation since these yield the changes in the dielectric constant induced by the strain component, and the dielectric constant determines the phase of reflected or transmitted light.

The photoelastic tensor is defined by the following relationship ${ }^{2}$ :

$$
\Delta\left(\underline{\epsilon}^{-1}\right)=\underline{p} \cdot \underline{e},
$$

where $\underline{\epsilon}$ is the tensor of the dielectric constant and $\underline{e}$ is the tensor of the applied strain. In the coordinate system where $\underline{\epsilon}$ is diagonal (without applied strain) the components of the dielectric tensor can be written as $^{3}$

$$
\Delta \epsilon_{i j}=-\epsilon_{i i} \epsilon_{j j} \sum_{k l} p_{i j k l} \Delta e_{k l}
$$

It is customary to introduce an abbreviated notation, since many of the elements are zero or equal. ${ }^{2}$ In this notation

$$
p_{m n}=-\frac{1}{2 \epsilon_{i i} \epsilon_{j j}} \frac{\partial \epsilon_{i j}}{\partial e_{k l}}\left(1+\delta_{k l}\right),
$$

where $m$ and $n$ represent $i j$ and $k l$ according to the rules $11 \rightarrow 1,22 \rightarrow 2,33 \rightarrow 3,23 \rightarrow 4,13 \rightarrow 5$, $12 \rightarrow 6$. In wurtzite structure only six independent photoelastic coefficients exist: $p_{11}=p_{12}, p_{12}=p_{21}$, $p_{13}=p_{23}, p_{31}=p_{32}, p_{33}, p_{44}=p_{55}$, and $p_{66}$ $=\frac{1}{2}\left(p_{11}-p_{12}\right)$; the remaining $p$ coefficients are

zero. From the induced change in $\epsilon$ given by (17) or (18) the change in phase can be computed,

$$
\begin{aligned}
\frac{\partial \theta}{\partial e_{k l}} & =\sum_{i j} \frac{\partial \theta}{\partial \epsilon_{i j}} \frac{\partial \epsilon_{i j}}{\partial e_{k l}} \\
& =\sum_{i j} \frac{\partial \theta}{\partial \epsilon_{i j}}\left(\frac{-2 \epsilon_{i i} \epsilon_{j j} p_{m n}}{\left(1+\delta_{k l}\right)}\right) .
\end{aligned}
$$

For small fluctuations we then have

$$
\begin{aligned}
\sigma^{2} & =\sum_{k l} \sigma_{e_{k l}}^{2}\left[\frac{\partial \Delta \theta_{x y}}{\partial e_{k l}}\right]^{2} \\
& =\sum_{k l} \sigma_{e_{k l}}^{2}\left(\frac{\partial \theta_{x}}{\partial e_{k l}}-\frac{\partial \theta_{y}}{\partial e_{k l}}\right)^{2},
\end{aligned}
$$

where $\sigma_{e_{k l}}$ is the standard deviation of the strain component $e_{e_{k l}}$ and (6) is applied. The problem of

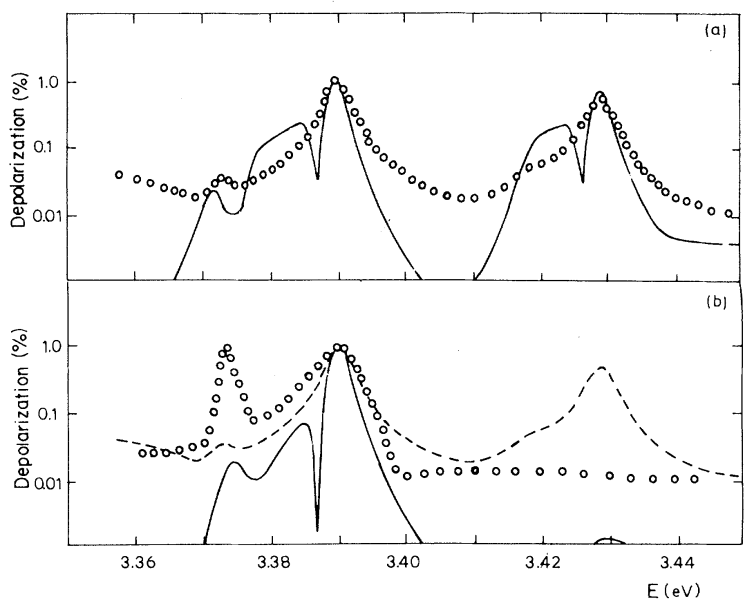

FIG. 2. Depolarization spectra for the reflected light in the exciton region of $\mathrm{ZnO}$ at $77 \mathrm{~K}$. The experimental spectra (dots) are taken from Ref. 4. The solid lines are spectra computed for $\overrightarrow{\mathrm{k}} \perp c$ and $\sigma_{e}=8.83 \times 10^{-5}$ (a) and for $\overrightarrow{\mathrm{k}} \| c$ and $\sigma_{e}=6.88 \times 10^{-5}(\mathrm{~b})$. In (b) the experimental spectrum from (a) is also shown (dashed line).

determining $\sigma^{2}$ is thus reduced to calculating the derivative $\partial \theta / \partial \epsilon_{i j}$ and the photoelastic coefficients $p_{m n}$. The computations of these are discussed in Appendixes A and B. The resulting expressions for $\partial \theta / \partial e_{k l}$ are also given in Appendix B.

\section{COMPARISON WITH EXPERIMENTAL RESULTS}

Experimental depolarization spectra for reflectivity due to excitations in $\mathrm{ZnO}$ have been measured by Filinski and Skettrup ${ }^{4}$ at 77 K. In Fig. 2 the depolarization ${ }^{4}$ defined in (15) is shown for geometries $\overrightarrow{\mathrm{k}} \perp c$ and $\overrightarrow{\mathrm{k}} \| c$ where $\overrightarrow{\mathrm{k}}$ is the wave vector of the incoming light and $c$ is the direction of the optical axis in $\mathrm{ZnO}$ (wurtzite structure). It is seen that the depolarization turns out to be most pronounced close to the minima of reflectivity. The crystals were selected to yield the smallest depolarization. The level of maximum depolarization of the crystals is only about $1 \%$ (corresponding to $\sigma=8^{\circ}$ ), and the expansion of (15) is thus valid. (In transmission spectra, however, up to ten times more depolarized than polarized light can be obtained in the spectral range of the Urbach absorption. $^{5,6}$ )

Letting the $c$ axis go along the $z$ direction and assuming all of the strain components to have the same standard deviation $\sigma_{e}$, we obtain from (15) and (20) 


$$
\begin{gathered}
\frac{\sigma^{2}}{2}=\frac{\sigma_{e}^{2}}{2}\left[\left(\frac{\partial \theta_{x}}{\partial e_{x x}}-\frac{\partial \theta_{z}}{\partial e_{x x}}\right]^{2}+\left(\frac{\partial \theta_{x}}{\partial e_{y y}}-\frac{\partial \theta_{z}}{\partial e_{y y}}\right)^{2}+\left(\frac{\partial \theta_{x}}{\partial e_{z z}}-\frac{\partial \theta_{z}}{\partial e_{z z}}\right)^{2}\right. \\
\left.+\left[\frac{\partial \theta_{x}}{\partial e_{x z}}-\frac{\partial \theta_{z}}{\partial e_{x z}}\right]^{2}+\left(\frac{\partial \theta_{z}}{\partial e_{y z}}\right]^{2}+\left[\frac{\partial \theta_{x}}{\partial e_{x y}}\right)^{2}\right] \text { for } \overrightarrow{\mathrm{k}} \perp c
\end{gathered}
$$

and

$$
\begin{aligned}
\frac{\sigma^{2}}{2} & =\frac{\sigma_{e}^{2}}{2}\left[\left(\frac{\partial \theta_{x}}{\partial e_{x x}}-\frac{\partial \theta_{y}}{\partial e_{x x}}\right]^{2}+\left(\frac{\partial \theta_{x}}{\partial e_{y y}}-\frac{\partial \theta_{y}}{\partial e_{y y}}\right)^{2}+\left(\frac{\partial \theta_{x}}{\partial e_{x y}}-\frac{\partial \theta_{y}}{\partial e_{x y}}\right)^{2}\right] \\
& =12 \sigma_{e}^{2}\left[\operatorname{Im}\left[\frac{1}{\sqrt{\epsilon_{x}}\left(1-\epsilon_{x}\right)}\left(K_{66}+2 C_{5} F_{66}\right)\right]^{2}\right] \text { for } \overrightarrow{\mathrm{k}} \| c .
\end{aligned}
$$

The derivatives are obtained from Appendix B. For $\overrightarrow{\mathbf{k}} \| c$ the three terms in (22) are similar, and the result is therefore expressed explicitly in terms of the quantities from Appendix B.

In order to compute the depolarization spectra (21) and (22) it is necessary to know the spectral behavior of the photoelastic coefficients in the exciton region. This has never been measured in that spectral range, and $p_{m n}$ must then be computed from the expressions given in Appendix B. However, only $p_{44}$ and $p_{66}$ can be computed, since the $K$ constants except for $K_{44}$ and $K_{66}$ are unknown. This means that only the spectrum (22) and the last two terms of (21) can be computed with known parameters. However, as described in Appendix C, it is possible to make a rough estimate of the other $K$ values so the remaining $p$ coefficients can be obtained. In this way all the photoelastic coefficients of $\mathrm{ZnO}$ can be computed throughout the whole exciton region. Hence, any question concerning the strain dependence of the exciton spectra of $\mathrm{ZnO}$ can, in principle, be answered.

The depolarization spectra obtained in this way from (21) and (22) are shown in Fig. 2. It is seen that a relatively good agreement with the experimental spectra is obtained. The relative magnitudes of the peaks, their positions and shapes, fit well with the experimental spectra. However, the strong peak near $3.37 \mathrm{eV}$ for $\overrightarrow{\mathrm{k}} \| c$ is not possible to reproduce with this theory of strain fluctuations. Also, the general background level of depolarization cannot be reproduced. Furthermore, the sharp minima of the theoretical curves are not seen in the experimental spectra. It can thus be concluded that there must be additional effects that cause depolarization. The strain distribution, however, implies spectra that reproduce the essential features, and such a distribution of local strain throughout the crystal volume are, therefore, likely to be one of the main reasons for the depolarization spectra obtained by Filinski and Skettrup. ${ }^{4}$ There is only one adjustable parameter in Fig. 2, the standard deviation $\sigma_{e}^{2}$ which just yields a shift along the $D$ axis. This value is obtained by shifting the spectra vertically to obtain fitting at the maximum at $3.39 \mathrm{eV}$. This procedure yields

$$
\sigma_{e}=\left\{\begin{array}{l}
6.88 \times 10^{-5} \text { for } \overrightarrow{\mathrm{k}} \| c \\
8.83 \times 10^{-5} \text { for } \overrightarrow{\mathrm{k}} \perp c .
\end{array}\right.
$$

These values are, as expected, almost equal. They are of the order $10^{2}-10^{3}$ times less than strains necessary for breaking the crystals. These relatively small values are thus sufficient to give depolarization levels up to $1 \%$ ( $8^{\circ}$ of standard deviation) in the region of excitonic resonance.

\section{ACKNOWLEDGMENTS}

The author wishes to thank Dr. I. Filinski for many stimulating discussions during the work.

\section{APPENDIX A}

We wish to obtain $\partial \theta / \partial \epsilon_{i j}$ for reflection spectra. Since the reflectivity can be written

$$
r_{\alpha} e^{i \theta_{\alpha}}=\frac{1-\left(\epsilon_{\alpha \alpha}\right)^{1 / 2}}{1+\left(\epsilon_{\alpha \alpha}\right)^{1 / 2}}
$$

the phase is given by

$$
\theta_{\alpha}=\operatorname{Im}\left[\ln \left(\frac{1-\left(\epsilon_{\alpha \alpha}\right)^{1 / 2}}{1+\left(\epsilon_{\alpha \alpha}\right)^{1 / 2}}\right)\right],
$$


where Im means imaginary part. Hence the derivative is given by

$$
\frac{d \theta_{\alpha}}{d \epsilon_{\alpha \alpha}}=\operatorname{Im}\left[-\frac{1}{\left(\epsilon_{\alpha \alpha}\right)^{1 / 2}\left(1-\epsilon_{\alpha \alpha}\right)}\right],
$$

where $\alpha$ represents either $x, y$, or $z$.

The derivatives with respect to off-axis elements of the dielectric tensor are more involved, since these imply not only changes in reflectivity, but also a change in the optical-axis direction for anisotropic crystals. We shall consider here the uniaxial case and only deal with the cases where the light propagates parallel $(\overrightarrow{\mathbf{k}} \| c)$ or perpendicular $(\overrightarrow{\mathrm{k}} \perp c)$ to the optic axis, corresponding to the experimental spectra shown in Fig. 2. We shall not go into detail with the computations of (A3) for off-axis elements. The method consists in diagonalizing the dielectric tensor by turning the coordinate system, the angle $\alpha$, given by

$$
\tan 2 \alpha=\frac{2 \epsilon_{i j}}{\epsilon_{i i}-\epsilon_{j j}} .
$$

The reflected light can then be written as

$$
\begin{aligned}
\left\{\begin{array}{l}
E_{r i} \\
E_{r j}
\end{array}\right\}= & \left\{\begin{array}{cc}
\cos \alpha & -\sin \alpha \\
\sin \alpha & \cos \alpha
\end{array}\right\}\left\{\begin{array}{cc}
r_{1} e^{i \theta_{1}} & 0 \\
0 & r_{2} e^{i \theta_{2}}
\end{array}\right\} \\
& \times\left\{\begin{array}{cc}
\cos \alpha & \sin \alpha \\
-\sin \alpha & \cos \alpha
\end{array}\right\}\left\{\begin{array}{l}
E_{I i} \\
E_{I j}
\end{array}\right\},
\end{aligned}
$$

where the reflectivities are found from (A1) with the eigenvalues of $\epsilon$ substituted for $\epsilon_{\alpha \alpha}$. It is then possible to obtain the derivative of the phase of reflectivity with respect to $\alpha$. After lengthy but straightforward computations one finds

$$
\frac{d \theta_{x}}{d \alpha}=\frac{r_{x}}{r_{z}} \sin \left(\theta_{x}-\theta_{z}\right)
$$

and

$$
\frac{d \theta_{z}}{d \alpha}=\frac{r_{z}}{r_{x}} \sin \left(\theta_{x}-\theta_{z}\right)
$$

when the light propagates along the $y$ direction.

The off-axis element $\epsilon_{13}$ is caused by the strain component $e_{13}$ [see Eq. (17)]. It turns out that there is a first-order dependence on $\alpha$ (and a second-order dependence on strain in the eigenvalues). Going through the computations outlined above yields the result (to first order)

$$
\frac{d \theta_{i}}{d \epsilon_{13}}=\frac{r_{i}}{r_{j}\left(\epsilon_{11}-\epsilon_{33}\right)} \sin \left(\theta_{1}-\theta_{3}\right),
$$

which is relevant for $\overrightarrow{\mathrm{k}} \perp c$.

The off-axis element $\epsilon_{12}$ is caused by the strain component $e_{12}$. It turns out that $\alpha=45^{\circ}$. There is now a first-order dependence on strain in the eigenvalues, but no angular dependence. The effect of this induced birefringence is that a phase change appears between two light beams reflected with polarization along the two main directions. The derivative of this induced phase is

$$
\begin{aligned}
\left(\frac{d \theta_{i}}{d \epsilon_{12}}\right]_{\epsilon_{12}=0} & =\left[\frac{d \theta_{i}}{d \lambda_{i}} \frac{d \lambda_{i}}{d \epsilon_{12}}\right]_{\epsilon_{12}=0}=\left[\operatorname{Im}\left(\frac{-1}{\sqrt{\lambda_{i}}\left(1-\lambda_{i}\right)}\right)(-1)^{i+1}\right]_{\epsilon_{12}=0} \\
& =\operatorname{Im}\left(\frac{-1}{\sqrt{\epsilon_{11}}\left(1-\epsilon_{11}\right)}\right)(-1)^{i+1} .
\end{aligned}
$$

\section{APPENDIX B}

We wish to compute $\partial \theta / \partial e_{k l}$ by means of (19) in order to obtain the standard deviation of the phase fluctuations in (20). It is then necessary to find $\partial \theta / \partial \epsilon_{i j}$ and $p_{m n} . \partial \theta / \partial \epsilon_{i j}$ is derived in Appendix A. The behavior of the photoelastic coefficients $p_{m n}$ of wurtzite structure close to the excitonic region has been discussed and derived by Berkowicz and Skettrup. ${ }^{7}$ We use their results here. In the notation of Ref. 7 (Appen$\operatorname{dix} \mathrm{C}$ ) and using the results of Appendix A, we then obtain

$$
\frac{\partial \theta_{x}}{\partial e_{x x}}=\frac{\partial \theta_{y}}{\partial e_{y y}}=\frac{\partial \theta_{x}}{\partial \epsilon_{x}}\left(-\epsilon_{x}^{2}\right) p_{11}=\operatorname{Im}\left(\frac{1}{\sqrt{\epsilon_{x}}\left(1-\epsilon_{x}\right)}\left(K_{11}+2 C_{5} F_{66}-2 C_{4} F_{1}+C_{2} G_{x}+C_{4} G_{x}^{\prime}\right)\right),
$$




$$
\begin{aligned}
& \frac{\partial \theta_{x}}{\partial e_{y y}}=\frac{\partial \theta_{y}}{\partial e_{x x}}=\frac{\partial \theta_{x}}{\partial \epsilon_{x}}\left(-\epsilon_{x}^{2}\right) p_{12}=\operatorname{Im}\left(\frac{1}{\sqrt{\epsilon_{x}}\left(1-\epsilon_{x}\right)}\left(K_{12}-2 C_{5} F_{66}-2 C_{4} F_{1}+C_{2} G_{x}+C_{4} G_{x}^{\prime}\right)\right) \\
& \frac{\partial \theta_{z}}{\partial e_{x x}}=\frac{\partial \theta_{z}}{\partial e_{y y}}=\frac{\partial \theta_{z}}{\partial \epsilon_{z}}\left(-\epsilon_{z}^{2}\right) p_{31}=\operatorname{Im}\left(\frac{1}{\sqrt{\epsilon_{z}}\left(1-\epsilon_{z}\right)}\left(K_{31}+2 C_{4} F_{3}+C_{2} G_{z}+C_{4} G_{z}^{\prime}\right)\right), \\
& \frac{\partial \theta_{x, y}}{\partial e_{z z}}=\frac{\partial \theta_{x}}{\partial \epsilon_{x}}\left(-\epsilon_{x}^{2}\right) p_{13}=\operatorname{Im}\left[\frac{1}{\sqrt{\epsilon_{x}}\left(1-\epsilon_{x}\right)}\left(K_{13}-2 C_{3} F_{1}+C_{1} G_{x}+C_{3} G_{x}^{\prime}\right)\right), \\
& \frac{\partial \theta_{z}}{\partial e_{z z}}=\frac{\partial \theta_{z}}{\partial \epsilon_{z}}\left(-\epsilon_{z}^{2}\right) p_{33}=\operatorname{Im}\left(\frac{1}{\sqrt{\epsilon_{z}}\left(1-\epsilon_{z}\right)}\left(K_{33}+2 C_{3} F_{3}+C_{1} G_{z}+C_{3} G_{z}^{\prime}\right)\right) \\
& \frac{\partial \theta_{x, y}}{\partial e_{x y}}=\frac{\partial \theta_{x, y}}{\partial \epsilon_{x y}}\left(-2 \epsilon_{x}^{2}\right) p_{66}=\mp \operatorname{Im}\left(\frac{2}{\sqrt{\epsilon_{x}}\left(1-\epsilon_{x}\right)}\left(K_{66}+2 C_{5} F_{66}\right)\right) \\
& \frac{\partial \theta_{z}}{\partial e_{x y}}=0, \\
& \frac{\partial \theta_{x}}{\partial e_{x z}}=\frac{\partial \theta_{x}}{\partial \epsilon_{x z}}\left(-2 \epsilon_{x} \epsilon_{z}\right) p_{44}=-2 \frac{r_{x}}{r_{z}} \sin \left(\theta_{x}-\theta_{z}\right) \operatorname{Re}\left(\frac{K_{44}+C_{6} F_{44} / 2 \sqrt{2}}{\left(\epsilon_{x}-\epsilon_{z}\right)}\right), \\
& \frac{\partial \theta_{z}}{\partial e_{x z}}=-2 \frac{r_{z}}{r_{x}} \sin \left(\theta_{x}-\theta_{z}\right) \operatorname{Re}\left(\frac{K_{44}+C_{6} F_{44} / 2 \sqrt{2}}{\left(\epsilon_{x}-\epsilon_{z}\right)}\right) .
\end{aligned}
$$

Here the indices 1,2 , and 3 were replaced by $x$, $y$, and $z$. The derivatives with respect to $e_{y z}$ are found from (B8) and (B9) by replacing $x$ with $y$. The real part of $p_{44} /\left(\epsilon_{x}-\epsilon_{z}\right)$ must be used in (B8) and (B9), since the imaginary part of $p_{44}$ will only contribute when multiplied with $\partial(\ln r) / \partial e_{x z}$ which is of second order and hence neglected.

\section{APPENDIX C}

We wish to make an estimate of the $K$ values of Eqs. (B1) - (B9) since only $K_{44}$ and $K_{66}$ are known from experiments. ${ }^{7}$ In order to do so, we use the fact that both $p_{44}$ and $p_{66}$ vanish at the same wavelength $[4328 \AA$ or $2.864 \mathrm{eV}$ at room temperature for $\mathrm{ZnO}$ (Ref. 7)]. This means that offdiagonal elements of the dielectric tensor are independent of strain at this wavelength. Furthermore, $p_{44}=p_{66}$ which is valid also for cubic symmetry. Hence, we apply the approximation of cubic symmetry at this wavelength. This implies that for $\lambda=4328 \AA$,

$$
\begin{aligned}
& p_{33}=p_{11}, p_{31}=p_{13}=p_{12}, \\
& p_{44}=p_{66}=\frac{1}{2}\left(p_{11}-p_{12}\right) .
\end{aligned}
$$

Further, since $p_{44}=p_{66}=0$, we have

$$
p_{11}=p_{12} \text {. }
$$

In this approximation there is then only one independent $p$ value at $4328 \AA$. That this approximation is relatively crude can be seen from the position ( $3945 \AA$ ) of the isotropic point for birefringence, which is not the same as that where $p_{44}=p_{66}=0$. Since the hexagonal crystal can be approximated by a quasicubic crystal with uniaxial stress along the [111] direction, ${ }^{8}$ the two isotropic points mentioned above are expected to be positioned at the same wavelength.

For the present purpose of computing 3 terms in (21), the $p$ values estimated need not be very accurate, so we apply the approximation (C1) and (C2).

TABLE I. Parameters used in calculations of photoelastic coefficients of $\mathrm{ZnO}$. The values are taken from Ref. 7, except for the energies and damping, which are valid for $T=77 \mathrm{~K}$ and derived by fitting to experimental reflectivity spectra.

\begin{tabular}{llll}
\hline \hline$E_{x 1}(\mathrm{eV})$ & 3.3695 & $\Gamma_{x}=\Gamma(\mathrm{meV})$ & 5.50 \\
$E_{x 2}(\mathrm{eV})$ & 3.3752 & $\Delta(\mathrm{meV})$ & 41 \\
$E_{x 3}(\mathrm{eV})$ & 3.4160 & $\lambda(\mathrm{meV})$ & -2.9 \\
$K_{1} P_{x x}^{1}\left(\mathrm{eV}^{2}\right)$ & 0.15 & $E_{1}(\mathrm{eV})$ & 3.4295 \\
$K_{1} P_{x x}^{2}\left(\mathrm{eV}^{2}\right)$ & 0.30 & $K_{2} P_{x}^{2}\left(\mathrm{eV}^{2}\right)$ & 2.704 \\
$K_{1} P_{z z}^{2}\left(\mathrm{eV}^{2}\right)$ & 0.0 & $K_{2} P_{z}^{2}\left(\mathrm{eV}^{2}\right)$ & 3.153 \\
$K_{1} P_{x x}^{3}\left(\mathrm{eV}^{2}\right)$ & 0.0 & $\epsilon_{\infty}(\overrightarrow{\mathrm{E}} \perp c)$ & 2.642 \\
$K_{1} P_{z z}^{3}\left(\mathrm{eV}^{2}\right)$ & 0.37 & $\epsilon_{\infty}(\overrightarrow{\mathrm{E}} \| c)$ & 2.524 \\
& & \multicolumn{1}{|c}{${ }^{2}$} & \\
\hline \hline
\end{tabular}


TABLE II. Deformation potentials and $K$ values for $\mathrm{ZnO}$, taken from (a) Langer et al. (Ref. 10), (b) Rowe et al. (Ref. 11), and (c) Berkowicz and Skettrup (Ref. 7).

\begin{tabular}{lcccccccc}
\hline \hline & $\begin{array}{c}C_{1} \\
(\mathrm{eV})\end{array}$ & $\begin{array}{c}C_{2} \\
(\mathrm{eV})\end{array}$ & $\begin{array}{c}C_{3} \\
(\mathrm{eV})\end{array}$ & $\begin{array}{c}C_{4} \\
(\mathrm{eV})\end{array}$ & $\begin{array}{c}C_{5} \\
(\mathrm{eV})\end{array}$ & $\begin{array}{c}C_{6} \\
(\mathrm{eV})\end{array}$ & $K_{44}$ & $K_{66}$ \\
\hline $\begin{array}{l}\text { Ref. a } \\
\text { Ref. b }\end{array}$ & -3.8 & -3.8 & -0.8 & +1.4 & -1.2 & -2.0 & & \\
Ref. c & & -4.0 & -1.2 & -0.9 & & & & \\
\hline \hline
\end{tabular}

Introducing $(\mathrm{C} 1)$ and $(\mathrm{C} 2)$ into the expressions for $p_{m n}$ (Ref. 7) at $\lambda=4328 \AA$, we obtain four equations for the five unknown $K$ values. The remaining equation can be obtained from experimental results of $\mathrm{Yu}$ and Cardona ${ }^{9}$ on the " $Q$ coefficients" which are combinations of the $p_{m n}$ values.

In Ref. 9 there are two sets of measurements $\left(Q_{33}-Q_{13}\right)$ and $\left.Q_{11}-Q_{31}\right)$ that can be used. It turns out that one obtains $K_{11}=-174$ from the $\left(Q_{33}-Q_{13}\right)$ value and $K_{11}=198$ from the other. These values only deviate by $12 \%$, but the two values have different signs. This is surprising because the approximations used above are not expected to be so bad that sign problems should arise. We have not found it worthwhile for the present purpose to pursue this sign problem further, but choose the positive sign since this keeps the $p$ values at the numerical lowest values in the exciton spectral range. We then get

$$
K_{11} \simeq K_{12} \simeq K_{13} \simeq 186
$$

and

$$
K_{31} \simeq K_{33} \simeq 193
$$

where the average value of the two $K_{11}$ values (with positive sign) have been used, and where $\left(\epsilon_{3} / \epsilon_{1}\right)^{2}$ $=1.0373$ at $4328 \AA$ is applied (value taken from Yu and Cardona $\left.{ }^{9}\right)$. Since $K_{66}=\frac{1}{2}\left(K_{11}-K_{12}\right), K_{11}$ and $K_{12}$ should deviate by $2 K_{66}=-3.5$, or $2 \%$. Hence this discrepancy is not of importance for the present purpose.

There is then sufficient information to compute the expected spectra of the photoelastic coefficients in the exciton region. The parameters of Tables $I$ and II and Eq. (C3) are used. In Table II, $C_{1}, C_{2}$, $C_{3}$, and $C_{4}$ from Ref. a are chosen, since these are consistent with the signs expected from a quasicubic model (see, e.g., Berkowicz and Skettrup ${ }^{7}$ ). $C_{5}, C_{6}$ and $K_{44}, K_{66}$ are taken from Ref. c, since these have been determined directly from fits to $p_{44}$ and $p_{66}$ at room temperature.
${ }^{1}$ M. Born and E. Wolf, Principles of Optics (Pergamon, New York, 1975).

2J. F. Nye, Physical Properties of Crystals (Oxford University Press, London, 1960).

${ }^{3}$ E. S. Kohn, J. Appl. Phys. 40, 2608 (1969).

${ }^{4}$ I. Filinski and T. Skettrup, in Proceedings of the 12th International Conference on the Physics of Semiconductors, Stuttgart, 1974, edited by M. H. Pilkuhn (Teubner, Stuttgart, 1974), p. 1222.

${ }^{5}$ I. Filinski and T. Skettrup, in Proceedings of the 13th International Conference on the Physics of Semiconductors, Rome, 1976, edited by F. G. Fumi (Marves, Rome, 1976), p. 861.
${ }^{6}$ T. Skettrup, Phys. Status Solidi B 103, 613 (1981).

${ }^{7}$ R. Berkowicz and T. Skettrup, Phys. Rev. B 11, 2316 (1975)

8J. J. Hopfield, J. Phys. Chem. Solids 15, 97 (1960).

${ }^{9}$ P. Y. Yu and M. Cardona, J. Phys. Chem. Solids 34, 29 (1973).

10P. W. Langer, R. N. Euwema, K. Eta, and T. Koda, Phys. Rev. B 2, 4005 (1970).

$11 \mathrm{~J}$. E. Rowe, M. Cardona, and F. H. Pollak, in Proceedings of the International Conference on II-VI Semiconducting Compounds, Brown University, 1967, edited by D. G. Thomas (Benjamin, New York, 1967), p. 112. 